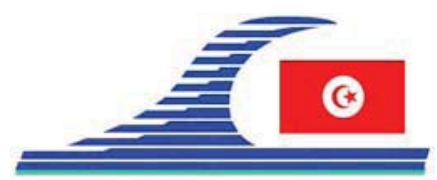

Conférence Méditerranéenne Côtière et Maritime

EDITION 1, HAMMAMET, TUNISIE (2009)

Coastal and Maritime Mediterranean Conference

Disponible en ligne - http://www.paralia.fr-Available online

\title{
Bilan de 25 ans de gestion d'une flèche sableuse à pointe libre
}

\author{
Hugues HEURTEFEUX ${ }^{1}$, François SABATIER ${ }^{2}$, \\ Stéphanie GROSSET ${ }^{1}$, Philippe RICHARD ${ }^{1}$
}

1. EID Méditerranée, 165 avenue Paul Rimbaud, 34184 Montpellier Cedex 4, France. hheurtefeux@eid-med.org

2. Aix-Marseille Université, CEREGE, Europôle de l'Arbois, BP80, 13545 Aix en Provence Cedex04, France.sabatier@cerege.fr

\section{Résumé :}

L'EID Méditerranée, opérateur public en zones humides, suit depuis plus de 20 ans, par des profils topographiques, l'évolution morphologique d'une flèche sableuse à pointe libre appelée «La Gracieuse », située à l'embouchure du Grand Rhône, face au Port Autonome de Marseille. Une analyse par corrélations des profils montre une forte indépendance entre la morphologie de la dune réhabilitée et de celle de la plage émergée sensu stricto (du pied de dune au trait de côte). Par ailleurs les résultats montrent une régularisation du trait de côte qui passe d'une allure convexe à rectiligne. Le fonctionnement de la flèche semble ainsi lié à la destruction d'un stock fossile en mer (ancien bras du Pégoulier) influençant surtout les limites émergées de l'enveloppe sableuse : poulier et plage. Les propositions d'aménagement se basent donc plus sur des apports sableux pour tenter des rééquilibrages.

Mots-clés : Flèche sableuse - Dune - Trait de côte - Apports sableux - Plage

\section{Introduction}

La flèche de la Gracieuse est une flèche sableuse à pointe libre située à l'embouchure du grand Rhône, en Méditerranée, à quelques kilomètres à l'ouest de Marseille. Comprise entre les longitudes $4^{\circ} 51^{\prime} \mathrm{E}$ et $4^{\circ} 57^{\prime} \mathrm{E}$ et les latitudes $43^{\circ} 20^{\prime} \mathrm{N}$ et $43^{\circ} 25^{\prime} \mathrm{N}$, elle s'étire sur presque $5 \mathrm{~km}$ en direction du nord-est. Cette langue de sable constitue une défense naturelle pour le Port Autonome de Marseille (PAM), installé juste en arrière. Depuis les années 1960, un certain nombre d'aménagement de génie côtier se sont succédés, avec notamment de l'échouage volontaire de barges pour incurver la partie distale de la flèche de façon à empêcher le comblement du chenal permettant l'accès aux installations portuaires. A la fin des années 1980, des techniques de génie écologique, ont permis de redonner de la consistance au corps sableux de la flèche par l'installation de pièges à sable éolien (au moyen de fascines type ganivelles) et re-profilage de la dune. La part de ces aménagements dans le bilan sédimentaire du système est pris en compte en analysant le système littoral depuis la dune jusqu'aux fonds de 20 mètres afin de montrer que le recul n'affecte pas uniquement le trait de côte.

$$
\text { DOI: } 10.5150 / \mathrm{cmcm} .2009 .052-7
$$



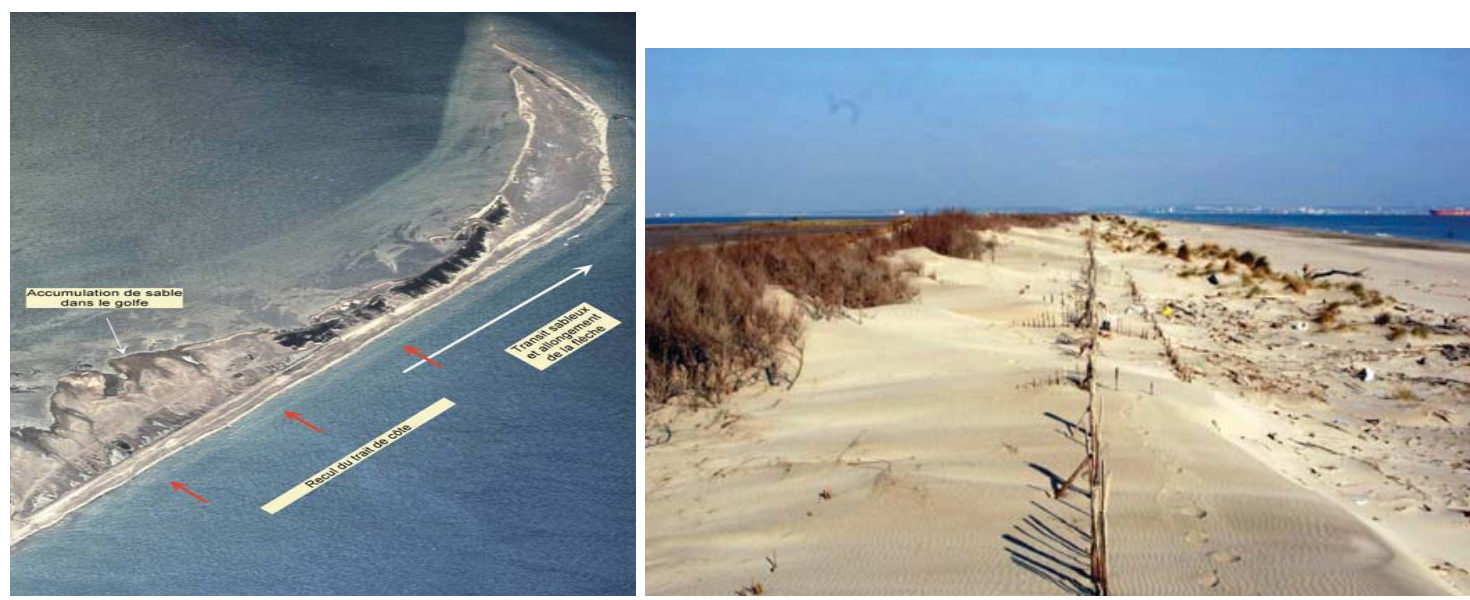

Figure 1. Situation de la Flèche de la Gracieuse.

\section{Fonctionnement à long terme}

Afin de définir le fonctionnement et le budget sédimentaire du rivage et de l'avant côte de la flèche de la Gracieuse à long terme, nous proposons un historique des variations du trait de côte et un modèle en boites basé sur des relevés bathymétriques séculaires. Cette démarche a déjà été entreprise (SUANEZ, 1997 ; BRUZZI, 1998 ; MAILLET, 2005 ; SABATIER et al., 2009a,) mais nous utilisons ici des données inédites et/ou peu publiées. La flèche de la Gracieuse connaît 3 zones aux évolutions continues: l'embouchure qui progresse en mer, la partie centrale qui s'érode et recule et le poulier qui s'engraisse et avance vers le NE (Fig. 2). Le budget sédimentaire dévoile d'importantes variations volumiques pour ces 3 zones probablement en relation avec les apports discontinus du Rhône. Sous l'effet de la dérive littorale dominante dirigée vers le NE, les sables érodés de la partie centrale de la flèche alimentent le Poulier. Cette évolution fait suite au changement de la position de l'embouchure du Rhône en 1892. En effet, cette dernière a été artificiellement déplacée vers l'ouest (Roustan) et l'on assiste depuis cette date à une modification progressive de la forme de la flèche d'une courbe vers une droite (Fig. 2). La régularisation du trait de côte est atteinte à partir des années 90 et sa relative stabilité s'explique probablement par la présence des ouvrages de reconstitution dunaire qui contribuent à fixer la position du système plage/dune (HEURTEFEUX \& RICHARD, 2005). Cependant la houle demeure particulièrement forte dans la partie centrale en raison de la morphologie sous marine héritée en lobe (présence de l'embouchure de Pégoulier jusqu'en 1892). Cette situation confère un état de fragilité préoccupant qui pourrait être masqué par l'apparente stabilité récente du trait de côte.

\section{Analyse de la topographie}

Un suivi littoral au moyen de profils topographiques est conduit depuis 1988 (figure 3). Une analyse fine sur la période 1989/1998 a été menée, en calculant pour chaque profil 
le volume total, de la partie dunaire et de la plage. Il en ressort que le volume total est fortement corrélé à celui de la plage $(\mathrm{R}=0,92)$ mais il ne montre pas de relation avec le volume de la dune $(\mathrm{R}=0,27)$, or c'est bien le bourrelet de dune qui est équipé en ganivelles.
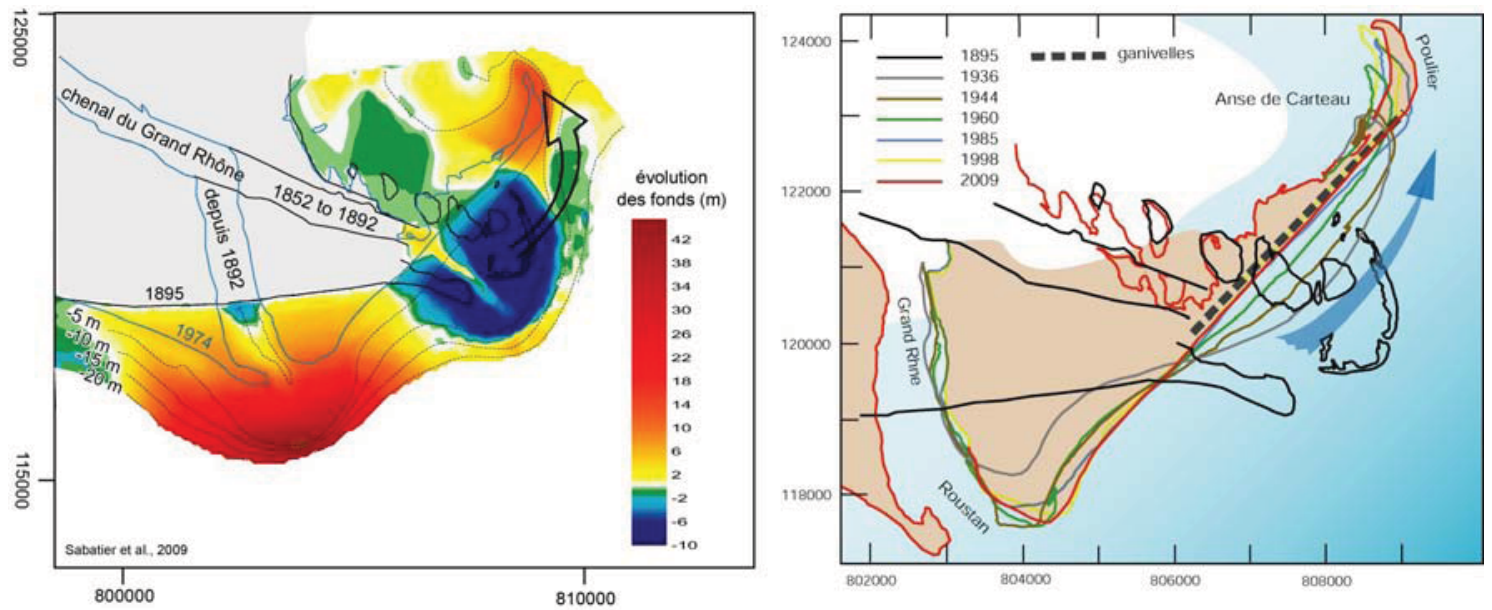

Figure 2. Evolution du trait de côte et des fonds durant le $20^{\text {ème }}$ siècle.

profil 1

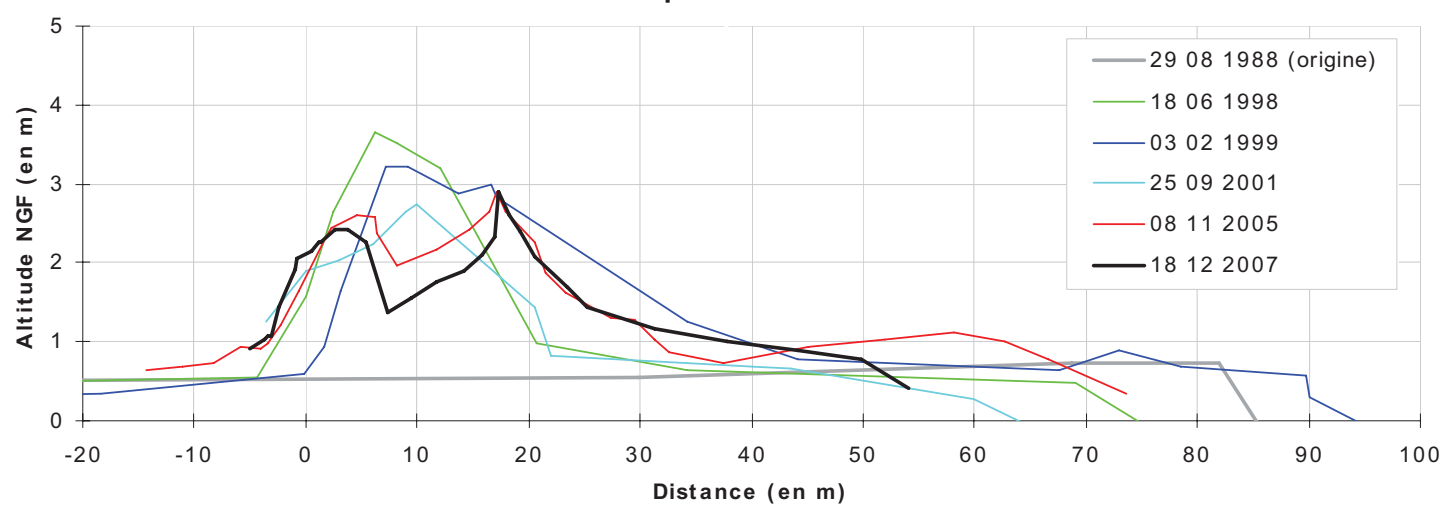

Figure 3. Evolution du profil de plage depuis l'origine des travaux de réhabilitation.

Une fois remplis de sable (surtout par l'action des vents de mer), les casiers de ganivelles donnent à la dune un volume de sable stable qui n'est plus sensible aux modifications morphologiques, alors que le secteur de la plage compris entre le pied de dune et le trait de côte lui reste complètement sensible. Logiquement, lorsque la distance entre la crête de dune et la position du trait de côte est la plus faible, le système subit les plus grandes pertes en sable par les houles de tempêtes. La plage, du pied de dune au trait de côte, constitue donc une zone tampon dont la largeur ne doit jamais être inférieure à $50 \mathrm{~m}$ (la distance optimale étant de $80 \mathrm{~m}$ ) pour le bon fonctionnement des ouvrages en ganivelles de la Gracieuse. La largeur optimale de cette zone tampon constitue aussi un fetch éolien indispensable à la formation des dunes (SABATIER et al., 2009b). Par contre la dégradation des ouvrages dès 2001 montre un effondrement 
puis un creusement central de la dune : le volume de la dune est alors reconnecté au fonctionnement global de la plage.

\section{Conclusions}

Un schéma global d'aménagement, incluant une nécessaire gestion des sédiments, est proposé pour l'ensemble de la flèche. Il hiérarchise différents degrés d'urgence en fonction de l'état de dégradation du cordon et de la plage. Des transferts terrestres de sédiments sont prévus depuis l'extrémité distale de la flèche jusque sur sa partie centrale. La dune sera revégétalisée avec des graminées (type Amophilia arenaria) ou des ligneux (type Tamarix gallica), des géotextiles biodégradables viendront aider la reprise végétale. Par endroit un maillage en ganivelles ou une simple ligne de mis en défens seront déployés. Le fonctionnement de la partie émergée de la flèche est hautement lié à l'action des houles qui viennent remanier le prodelta fossile du Pégoulier. Les actions menées jusqu'alors ont été en partie efficaces, mais ont montré une durée de vie limitée (20 ans pour les ouvrages de réhabilitation dunaire). De nouvelles opérations sont donc à envisager pour rééquilibrer les transferts naturels de sable depuis le Poulier de la flèche. Mais, comme souvent en matière de gestion du littoral, il s'agira de continuer à suivre ces processus pour mener à temps les indispensables mesures d'entretien.

\section{Références bibliographiques}

BRUZZI C. (1998). Les tempêtes et l'évolution morphosédimentaire des plages orientales du delta du Rhône. Thèse de doctorat, Université de Provence, UFR des Sciences Géographiques et de l'Aménagement, Aix-en-Provence, 325 p.

HEURTEFEUX H., RICHARD P. (2005). Vingt ans de protection des dunes littorales par des techniques douces : bilan et perspectives à travers quelques exemples en Méditerranée française. Milieux littoraux, Paris, Harmattan, pp 143-166.

MAILLET G.-M. (2005). Relations sédimentaires récentes et actuelles entre un fleuve et son delta en milieu microtidal : Exemple de l'embouchure du Rhône. Thèse de doctorat, Université de Provence, Aix-Marseille 1, 301 p.

SABATIER F, SAMAT O, ULLMANN A, SUANEZ S. (2009a). Connecting largescale coastal behaviour with coastal management of the Rhone delta. Geomorphology, 107, pp 79-89.

SABATIER F., ANTHONY E., HEQUETTE A., SUANEZ S., MUSEREAU J., RUZ M-H., REGNAULD H. (2009b). Morphodynamics of beach/dune systems: examples from the coast of France. Géomorphologie : reliefs, processus et environnements, 1, pp 3-22.

SUANEZ S. (1997). Dynamiques sédimentaires actuelles et récentes de la frange littorale orientale du delta du Rhône. Thèse de doctorat, Université de Provence, Aixen-Provence, $282 \mathrm{p}$. 